\title{
Application of the Sine-Cosine Algorithm to the Optimal Design of a Closed Coil Helical Spring
}

\author{
M. A. Rodriguez-Cabal (D); L. F. Grisales-Noreña (D); C. A. Ramírez-Vanegas (D); and A. Arias-Londoño (DD) \\ DOI: https://doi.org/10.32397/tesea.vol2.n2.5 \\ Research paper \\ Received: 1 September 2021; Accepted: 2 December 2021; Published: 18 January 2022
}

\begin{abstract}
This paper proposes the application of the sinecosine algorithm (SCA) to the optimal design of a closed coil helical spring. The optimization problem addressed corresponds to the minimization of total spring volume subject to physical constraints that represents the closed coil helical spring such as maximum working load, shear stress, and minimum diameter requirements, among other. The resulting mathematical formulation is a complex nonlinear and non-convex optimization model that is typically addressed in literature with trial and error methods or heuristic algorithms. To solve this problem efficiently, the SCA is proposed in this research. This optimization algorithm belongs to the family of the metaheuristic optimization techniques, it works with controlled random processes guided by sine and cosine trigonometric functions, that allows exploring and exploiting the solution space in order to find the best solution to the optimization problem. By presenting as main advantage an easy implementation at any programming language using sequential quadratic programming; eliminating the need to uses specialized and costly software. Numerical results demonstrating that the proposes SCA allows reaching lower spring volume values in comparison with literature approaches, such as genetic algorithms, particle swarm optimization methods, among others. All the numerical simulations have been implemented in the MATLAB software.
\end{abstract}

Index Terms-Mechanical analysis; machine elements design; sine-cosine algorithm; nonlinear optimization model; closed coil helical spring.

\section{INTRODUCTION}

$\mathbf{T}$ HE design of mechanical elements for industrial machinery is a complex and delicate task, since various factors must be considered for its construction, such as: the efforts to which the part will be subjected, the type of material to be used, the correct geometry for the coupling of the elements, among others [1]. Finding an adequate solution to the different mechanical design problems requires time to simulate, manufacture and validate the different prototypes associated with the part under analysis. This leads to an arduous and long process,

Miguel Angel Rodriguez-Cabal es estudiante de la maestría en gestión energética del Instituto Tecnologico Metropolitano, Medellin 050013, Colombia; (email: miguelrodriguez220490@correo.itm.edu.co)

Luis Fernando Grisales-Noreña, Doctor en ingeniería, Docente adscrito al Instituto Tecnológico Metropolitano, Medellín 050013, Colombia; (email: luisgrisales@itm.edu.co)

Carlos Alberto Ramírez-Vanegas, Facultad de Ciencias Básicas, Universidad Tecnológica de Pereira, Pereira 660003, Colombia; (email: caramirez@utp.edu.co)

Andrés Arias-Londoño, Facultad de Ingeniería, Doctor en ingeniería, Docente adscrito a la Institución Universitaria Pascual Bravo, Medellín 050036, Colombia; (email: andres.arias366@pascualbravo.edu.co) in which time and financial resources must be invested [2]. The latter being the greatest limitation, since in many cases companies do not have the resources to perform mechanical resistance tests on the elements they design. In the industrial field, mechanical design is important to provide quality and safety in the manufacture of the components associated with the different machines and equipment [3]. Mechanical design can be divided into various stages: In the first stage, a preliminary or conceptual design of the piece begins, in addition, a vision of what is wanted is given with the necessary measures. In the second stage, the simulation and analysis are carried out, in which it is guaranteeing that the element will be able to withstand the loads and stresses subjected, then the prototyping is carried out to validate experimentally the piece before to make the manufacture process; this ensures that the element will not be affected by premature failure [4]. The first and second parts are responsibility of the designer, in this way, the correct selection of materials and dimensions of the element will depend on its manufacture cost and its reliability. It is for the above that, various strategies have been implemented for designing machine elements, by searching the most appropriate solution for the design problem by means of optimization techniques. These techniques perform the minimization or maximization of an objective function, starting from a defined solution space by a set of constraints; so that from a mathematical model and the input parameters it is possible to determine a good solution, with the aim of reducing calculation times and improves the quality of the solution compared to conventional design methods. An example of optimal mechanical design, is the case presented by [5], where the geometry of a stepped transmission shaft was optimized by means of a continuous genetic algorithm (GA), using the weight of the shaft as the objective and the maximum deflection as a set of restrictions and the difference between the diameters, this with respect to the coupling of the transmission devices; finally, the authors validated the simulation results with specialized design software by demonstrating that the solution achieves represents an adequate solution to the problem analysed. On the other hand, in [6] the authors implementing a Rao algorithms to solve different engineering optimization problems such as the design of a clutch disc, bearings, tube exchanger, and casing, among others. Demonstrating that the proposed method obtaining good solutions compared with other conventional algorithms, in terms of computational time and quality of the solution. A similar work is presented by 
[7], where the authors perform the optimization of different engineering problems by using multiples objective functions, such as: minimizing the weight of a spring, minimizing the weight of a gear train, minimizing the weight of a welded beam, and minimizing the volume of a tank. The solution technique proposed by the authors is known as the chaotic gray wolf, the solution method is compared with other articles reported in the literature by demonstrating that the proposed algorithm presented a good performance in comparison with these; the authors do not report the times from the solution. Another case is presented in [8], by using a multi objective optimization for designing a helical spring, taking as objective functions the minimization of the weight of the element and the natural frequency. The set of constraints used both the physical characteristics and forces that must support the spring (as the dynamic constraints associated with load variation). To solve this problem, the authors implemented a genetic algorithm, a method based on sequential programming (active set), interior point method, and a pattern search algorithm, where it is highlighted that the last two do not perform a total exploration of the solution space, so it can fall into local optimal solutions. The authors report that the best solution is founded by the Active set, but they do not compare processing times.

These optimization cases make it clear that performing the optimization of a complete machine is a complex task, is for this reason that the literature is focused on the constructive elements that are more prone to failure or that are widely used regardless of the final application. Among these elements are closed coil helical springs, which are used in suspensions, damping systems, among others applications, this element is subjected to different compression and traction forces by supporting different forces and loads, both static and dynamic, therefore that the correct operation of the device to which it is attached depends on its correct sizing [9], [10]. It is for the above that in the present work the minimization of the volume of a closed coil helical spring is proposed and as a solution method the application of the Sine and Cosine algorithm which has presented good performance in terms of the quality of the solution and computational times in other mechanical applications.

Finally, the document is organized as follows: Section [?] presents the mathematical formulation of the optimal design of a closed coil helical spring. In section [?] the solution technique is explained. Section [?] submits the results of the algorithms and a comparison between them in terms of the quality of the solution. Finally, conclusions and future work are presented.

\section{Mathematical Modeling}

In this section is presented the general nonlinear and non-convex optimization model for closed coil helical spring. This mechanical component is depicted in Fig. 1. where two variables are illustrated, i.e., $d$ is the diameter of spring wire and $D$ is the mean coil diameter of the spring, both measured in centimeters. Note that the main goal in this optimization model is to minimize the volume of a closed coil helical

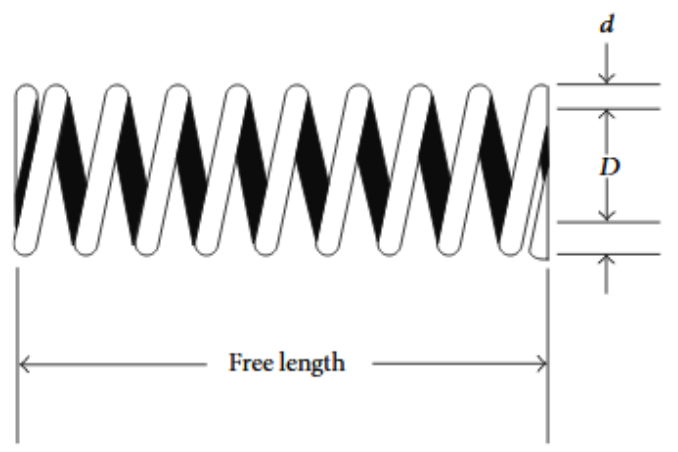

Fig. 1. Schematic drawing of a closed coil helical spring

spring considering several constraints. The mathematical optimization model is completely described as follows:

The objective function regarding the total volume of the spring is defined in Equation (1), where $V$ is the objective function value and $N_{c}$ represents the number of active coils.

$$
V=\left(\frac{\pi}{2}\right)^{2}\left(N_{c}+2\right) D d^{2}
$$

The shear stress must be less than the specified value and can be formulated as presented in Equation (2).

$$
\pi d^{3} S-8 C_{f} F_{\max } D \geq 0,
$$

where $F_{\max }$ is the maximum working load, $S$ is the permissible shear stress, and $C_{f}$ is defined as follows:

$$
C_{f}=\frac{4 C^{2}+1.46 C-2.46}{4 C(C-1)}, \text { being } C=\frac{D}{d} .
$$

Regarding the configuration of the spring, it is worth mentioning that the free length must be less than a specified value. The spring constant $K$ can be defined as presented Equation (3), where $G$ is the shear modulus.

$$
K=\frac{G d^{4}}{8 N_{c} D^{3}}
$$

Note that the deflection under maximum working load can be calculated as defined in Equation (4).

$$
\delta_{l}=\frac{F_{\max }}{K},
$$

It is assumed that the free length of the spring under the maximum working load is $\alpha$ times the solid length. Based on this assumption, the free length is given by (5).

$$
l_{f}=\delta_{l}+\alpha\left(N_{c}+2\right) d,
$$

which implies, that the maximum length admissible for the spring correspond to the mathematical expression presented in (6), where $l_{\max }$ is a design parameter.

$$
l_{\max }-l_{f} \geq 0,
$$


The wire diameter of the spring must be overpass a minimum specified value $d_{\min }$, which can be expressed mathematically as presented in Equation (7).

$$
d-d_{\min } \geq 0
$$

In addition, the outside diameter of the coil can not exceed a maximum specified which is defined in Equation (8).

$$
D_{\max }-D-d \geq 0 \text {. }
$$

Based on the physical characteristics of the spring the diameter of the coil must be at least $\beta$ times the diameter of the spring to ensure that the spring is not tightly wound. This physical constraint is formulated as follows:

$$
C-\beta \geq 0 .
$$

In the preload operation the deflection of the spring must be less than a specified quantity. The deflection of this condition can be expressed as 10 .

$$
\delta_{p}=\frac{F_{p}}{K}
$$

which implies that:

$$
\delta_{p \max }-\delta_{p} \geq 0 .
$$

Observe that the combined deflection must be consistent with the length and this can be formulated as presented in Equation (12).

$$
l_{f}-d_{p} \geq 0,
$$

The deflection from preload to maximum load must be equal to the design value as defined in Equation (13), being $\delta_{\omega}$ a specified constant.

$$
F_{\max }-F_{p}-K \delta_{\omega} \geq 0,
$$

Finally, to solve efficiently the studied mathematical model (11) to (13), the variables $d, D$ and $N_{c}$ are constrained with box-type conditions as defined in Expression (14).

$$
\begin{gathered}
d_{\min } \leq d \leq d_{\max }, \\
D_{\min } \leq D \leq D_{\max }, \\
N_{c \text { min }} \leq N \leq N_{c \max } .
\end{gathered}
$$

The parameters considering for the design of the closed coil helical spring are reported in Table I] These parameters were taken from [11].

\section{Solution TECHNiQue}

The mathematical optimization model presented from equations (1) to (14) is a complex nonlinear and non-convex optimization problem, with continuous variables and a single objective function. Due to the products and divisions between decision variables is not possible to proposed a convex reformulation that allows reaching the global optimum. However, this problem can be solved with combinatorial optimization techniques that allows dealing with the constraints via penalty factors added to the objective function [12]. To address this
TABLE I

CONDITIONS OF THE DESIGN OF THE CLOSED COIL HELICAL SPRING

\begin{tabular}{ccc}
\hline Parameter & Value & Unit \\
\hline$F_{\max }$ & 453.6 & $\mathrm{~kg}$ \\
$G$ & 808543.6 & $\mathrm{kgf} / \mathrm{cm}^{2}$ \\
$l_{\max }$ & 35.56 & $\mathrm{~cm}$ \\
$\delta_{\omega}$ & 3.175 & $\mathrm{~cm}$ \\
$d_{\max }$ & 1.016 & $\mathrm{~cm}$ \\
$D_{\max }$ & 7.620 & $\mathrm{~cm}$ \\
$N_{c \max }$ & 25 & - \\
$S$ & 13288.02 & $\mathrm{kgf} / \mathrm{cm}^{2}$ \\
$\alpha$ & 1.05 & - \\
$\beta$ & 3 & - \\
$d_{\min }$ & 0.508 & $\mathrm{~cm}$ \\
$D_{\min }$ & 1.270 & $\mathrm{~cm}$ \\
$N_{c \min }$ & 15 & -
\end{tabular}

nonlinear optimization problem in this research we propose the application of the sine-cosine algorithm (SCA) [13], which is completely described below.

The SCA is a methaeuristic optimization technique from the family of the particle swarm optimization methods that takes the advantages of the trigonometric sine and cosine function to explore and exploit the solution space [14], [15]. The SCA has been applied in different optimization problems such as: optimal power flow analysis [16], [17], feature selection in machine-learning algorithms [18], numerical optimization with nonlinear non-convex functions [19], [20], among other; but does not has been proposed for solving the problem addressed in this paper, for which this is one of the mains contributions of this research.

The main characteristics of the SCA for nonlinear optimization are presented below.

\section{A. Fitness function}

The main advantage of using metaheuristic optimization methods is the possibility of working in the infeasible solution space to analyze some promissory regions of this space. This relaxation can be made by using penalty factors in the objective function, which transforms it into a fitness function [17]. In the studied optimization problem, the proposed fitness function is defined in (15):

$z_{f}=\left(\begin{array}{l}V-\theta_{1} \min \left\{0, \pi d^{3} S-8 C_{f} F_{\max } D\right\}- \\ \theta_{2} \min \left\{0, l_{\max }-l_{f}\right\}-\theta_{3} \min \left\{0, d-d_{\min }\right\}- \\ \theta_{4} \min \left\{0, D_{\max }-D-d\right\}-\theta_{5} \min \{0, C-\beta\}- \\ \theta_{6} \min \left\{0, \delta_{p \max }-\delta_{p}\right\}-\theta_{7} \min \left\{0, l_{f}-d_{p}\right\}- \\ \theta_{8} \min \left\{0, F_{\max }-F_{p}-K \delta_{\omega}\right\}\end{array}\right)$

where $\theta_{1}, \ldots, \theta_{8}$ are penalty factors that allow controlling the impact of exploring the infeasible solution space, being all of them positive real numbers. Here we select for each component a value of 100 . Note that the fitness function can be equal to the objective function (1) if the solution space is totally feasible, i.e., all the constraints are satisfied.

Remark 1. The constraints regarding upper and lower bounds of the decision variables $d, D$ and $N_{c}$ defined in (14) are guarantee in the generation of all the potential solutions, which implies that all of them are always feasible [11]. 


\section{B. Initial population}

The SCA works with a population that evolves through the solution spaces by using a random controlled procedure [21]. The initialization of the proposed optimization algorithm is presented as follows:

$$
X^{t}=\left[\begin{array}{ccc}
x_{11} & x_{12} & x_{13} \\
x_{21} & x_{22} & x_{23} \\
\vdots & \vdots & \ddots \\
x_{n 1} & x_{n 2} & x_{n 3}
\end{array}\right],
$$

where $n$ represents the number of individuals considered in the population. Note that $X^{t}$ represents the population at the iteration $t, x_{i 1}$ represents the variable $d, x_{i 2}$ corresponds to the variable $D$, and $x_{i 3}$ is associated with the variable $N_{c}$.

To guarantee the upper and lower bound of each decision variable as defined in Equation (14), the following generation rule the $x_{i j}$ components in the initial population is considered:

$x_{i j}=x_{j}^{\min }+r_{1}\left(x_{j}^{\max }-x_{j}^{\min }\right) \forall j=1,2,3, \forall i=1,2, \ldots, n ;$

where $i j$ represents the row $i$ and column $j$ in the matrix of the initial population and $r_{1}$ is a random number, i.e., $r_{1} \in[0,1]$.

\section{Evolution criterion}

The SCA optimization algorithm evolves by considering a simple trigonometric rule. For doing so, let us consider that the fitness function for all individuals contained in $X^{t}$ has been evaluated, then, the best individual of that population is named $x_{\text {best }}$, Based on the best current solution, we select a possible new candidate $y^{t+1}$ or $x^{t+1}$ that will replace $X^{t}$ is some conditions are fulfilled:

$y_{i}^{t+1}=x_{i}^{t}+r_{2} \sin \left(r_{3}\right)\left|r_{4} x_{\text {best }}-x_{i}^{t}\right|, i=1,2, \ldots, n$, if $\delta \geq \frac{1}{2}$,

$z_{i}^{t+1}=x_{i}^{t}+r_{2} \cos \left(r_{3}\right)\left|r_{4} x_{\text {best }}-x_{i}^{t}\right|, i=1,2, \ldots, n$, if $\delta<\frac{1}{2}$,

being $r_{3}$ and $r_{4}$ random numbers between 0 to 1 and $-\pi$ to $\pi$, respectively, and $r_{2}$ is entrusted of the convergence of the algorithm, which can be calculated by using the equation 20]. Where $t_{\max }$ is the maximum number of iterations projected for the optimization process.

$$
r_{2}=1-\frac{t}{t_{\max }},
$$

Observed that $y_{i}^{t+1}$ and $z_{i}^{t+1}$ are the potential individuals that will replace $X_{i}^{t}$. This substitution can be made as follows: Select $y_{i}^{t+1}$ as a potential solution, if $z_{f}\left(y_{i}^{t+1}\right)<z_{f}\left(z_{i}^{t}\right)$; Select $z_{i}^{t+1}$ as a potential solution, if $z_{f}\left(z_{i}^{t+1}\right)<z_{f}\left(y_{i}^{t}\right)$; otherwise $X_{i}^{t+1}=X_{i}^{t}$. It is important to point out that if one component of $X_{i}^{t+1}$ does not fulfill its upper and lower bounds defined in (14), then, this potential individual is adjusted using (17) in order to preserve the feasibility of the current population during the entire optimization procedure.

\section{Finalization of the searching process}

The searching process of the SCA stops if one of the following criteria is fulfilled.

- If the maximum number of iteration is attained.

- If during $k_{\max }$ consecutive iterations the fitness does not exhibit any improvement,

\section{E. Algorithm implementation of the SCA}

Finally, Algorithm 1 presents the steps required bu the SCA for the optimal design of closed coil helical spring.

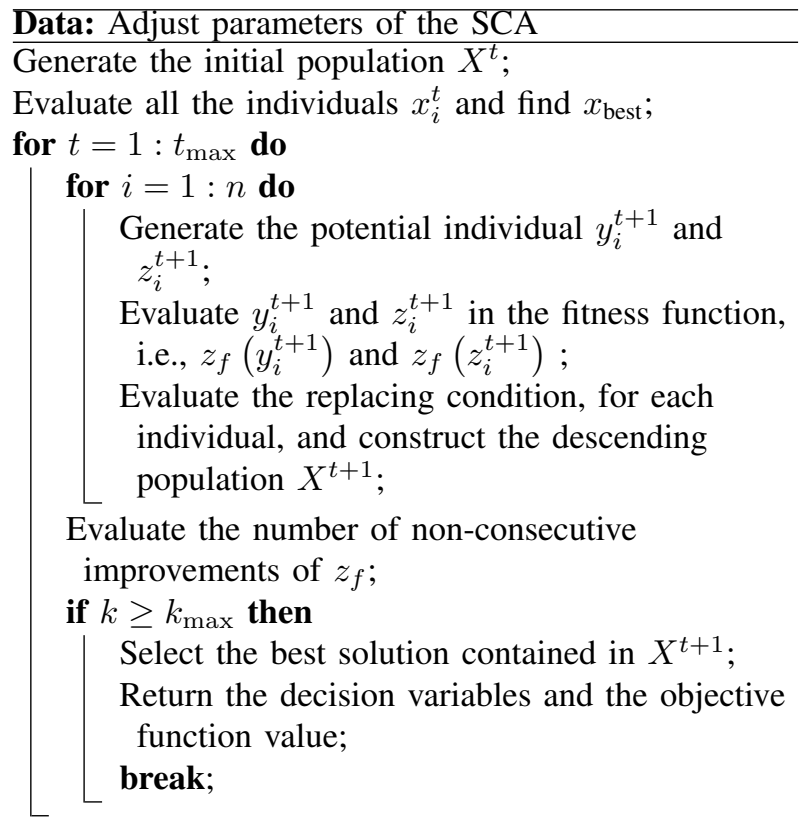

Result: Impress results

Algorithm 1: Proposed SCA for optimal designing of the closed coil helical spring

\section{Computational VAlidation}

The computational validation of the proposed SCA for the volume minimization of a closed coil helical spring is made in a desk-computer with an INTEL(R) Core(TM) $i 7-7700$ processor at $3.60 \mathrm{GHz}, 8 \mathrm{~GB}$ RAM, running a 64-bits in Windows 10 environment. MATLAB programming software version $2017 b$ was employed to implement the optimization procedure. To compare numerical results obtained by the solution method proposed, different optimization algorithm published in [11] have been employed. These optimization methods are: Classical tuning method (conventional), genetic algorithms (GA), particle swarm optimization (PSO), artificial bee colony $(\mathrm{ABC})$, teaching-based learning optimization (TBLO), and differential teaching-based learning optimization (DTBLO).

Figure 2 is presented the objective function performance for all the comparative methods and the proposed SCA. 


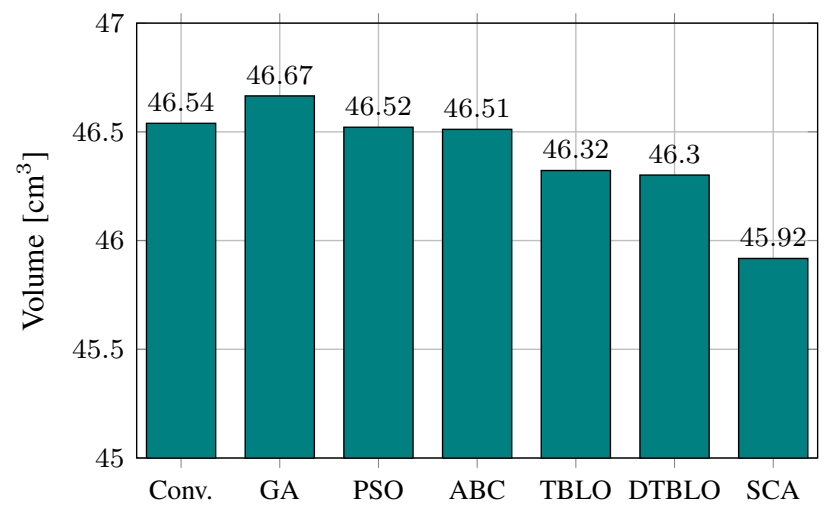

Fig. 2. Final volume of the closed coil helical spring with different metaheuristics

From results in Figure 2 we can observed that:

$\checkmark$ When the GA is compared with the conventional approach, it is possible to see that it has worse numerical performance, this situation can be attributable to the tuning of the searching parameters in the GA method, which have affected negatively its performance.

$\checkmark$ The proposed SCA improves the best objective function value reported in literature by the DTBLO method, since it passes from $46.30 \mathrm{~cm}^{3}$ to $45.92 \mathrm{~cm}^{3}$,i.e., an improvement about $0.83 \%$.

$\checkmark$ To reach the results reported by our proposed SCA algorithm was used 1000 individuals in the population, 10000 iterations, which takes about $70 \mathrm{~s}$ to optimize this problem, which finds the following final values for the decision variables, i.e., $d=0.673914 \mathrm{~cm}, D=$ $2.403081 \mathrm{~cm}$ and $N_{c}=15.051533$.

Figure 3 presents the histogram of the proposed SCA after 100 consecutive evaluations. Observe that there is 72 solutions in this histogram with values lower than the best solution reported by the DTBLO, which implies that our approach has a $72 \%$ of probabilities to improve that solution in only one execution. In addition, there are also 10 solutions with objective function values between $45.9 \mathrm{~cm}^{3}$ and $46 \mathrm{~cm}^{3}$, which implies that all of them are the better solutions reported in the literature at this moment in relation to the optimization of the closed coil helical spring, which is a clear contribution to the state-of-the-art in the area of mechanical components' design.

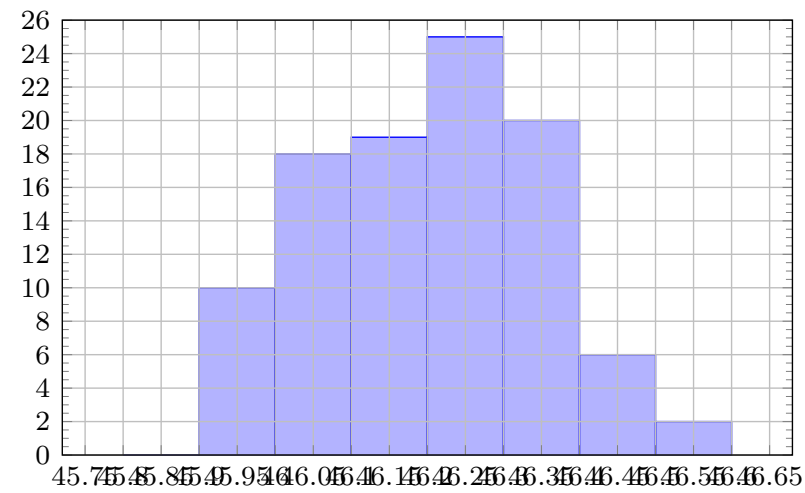

Fig. 3. Behavior of the SCA after 100 consecutive iterations

\section{Conclusions}

In this article, the optimal design of a closed coil helical spring was carried out via the sin-cosine algorithm (SCA). As objective function, the minimization of spring volume through an adequate selection of the internal diameter, wire diameter, and the number of active coils, by using as a set of constraints the physical conditions that should be considered in the design of helical springs that ensure a safe and reliable design. As comparison methods were employed the GA, PSO, ABC, TBLO and DTBLO proposed in the literature for solving the problem addressed in this paper, in terms of quality of the solution.

Computational simulations show that the proposed SCA approach obtains the best result in terms of objective function (volume of the helical spring), being $0.83 \%$ better than DTBLO method which corresponds to the best objective function value reported in literature taking only $70 \mathrm{~s}$ to find it. In addition, the statistical behavior of the SCA algorithm has demonstrated that in a high percentage of evaluations (i.e., $72 \%$ ) the solution reached by it is indeed better that all the known literature reports, which clearly confirmed the efficiency and robustness of this to deal with complex non-linear and non-convex optimization problems with low computational effort.

As future works it will be possible to make the following: i) to extend the studied SCA to mechanical design problems such as optimization of transmission shafts or optimal pulley design; and ii) to apply new metaheuristic approaches to the problem of the optimal design of the closed coil helical spring such as vortex search algorithm and hurricane-based optimizer in order to improve the quality of the solution reported in this research. The construction of the transformer comprises 3 main steps: the core, the windings, and their assembly. Figure 1 depicts a flow diagram that contains an overview of the construction and testing of the device, in which those 3 steps are in yellow, blue, and red, respectively.

\section{REFERENCES}

[1] G. P. Garcia, "Una teoría general de análisis en el diseño de elementos de máquinas," Ingeniería e Investigación, vol. 0, no. 13, pp. 31-42, 2010.

[2] M. A. Rodriguez-Cabal, J. A. Marín, L. F. Grisales-Noreña, O. D. Montoya, and J. A. S. Del Rio, "Optimization of a drive shaft using PSO algorithm," WSEAS Transactions on Applied and Theoretical Mechanics, vol. 13, pp. 130-139, 2018.

[3] R. G. Budynas and J. K. Nisbett, Shigley's Mechanical Engineering Design. New York: McGRAW-HILL, 9 ed., 2011.

[4] S. Bhaumik, R. Rangaraju, M. Parameswara, M. Venkataswamy, T. Bhaskaran, and R. Krishnan, "Fatigue failure of a hollow power transmission shaft," Engineering Failure Analysis, vol. 9, pp. 457-467, aug 2002.

[5] M. A. Rodriguez-Cabal, L. F. Grisales-Noreña, J. G. Ardila-Marín, and O. D. Montoya-Giraldo, "Optimal design of transmission shafts: a continuous genetic algorithm approach," Statistics, Optimization \& Information Computing, vol. 7, dec 2019.

[6] H. N. Ghafil and K. Jármai, "Dynamic differential annealed optimization: New metaheuristic optimization algorithm for engineering applications," Applied Soft Computing, vol. 93, p. 106392, aug 2020.

[7] M. Kohli and S. Arora, "Chaotic grey wolf optimization algorithm for constrained optimization problems," Journal of Computational Design and Engineering, vol. 5, pp. 458-472, mar 2017.

[8] M. Taktak, K. Omheni, A. Aloui, F. Dammak, and M. Haddar, "Dynamic optimization design of a cylindrical helical spring," Applied Acoustics, vol. 77, pp. 178-183, mar 2014. 
[9] L. Wu, L. Chen, H. Fu, Q. Jiang, X. Wu, and Y. Tang, "Carbon fiber composite multistrand helical springs with adjustable spring constant: design and mechanism studies," Journal of Materials Research and Technology, vol. 9, pp. 5067-5076, may 2020.

[10] J. Ke, Z. yu Wu, Y. sheng Liu, Z. Xiang, and X. dong Hu, "Design method, performance investigation and manufacturing process of composite helical springs: A review," Composite Structures, vol. 252, p. 112747 , nov 2020 .

[11] B. Thamaraikannan and V. Thirunavukkarasu, "Design Optimization of Mechanical Components Using an Enhanced Teaching-Learning Based Optimization Algorithm with Differential Operator,' Mathematical Problems in Engineering, vol. 2014, pp. 1-10, 2014.

[12] L. F. Grisales-Noreña, O. D. Garzón-Rivera, J. A. Ocampo-Toro, C. A. Ramos-Paja, and M. A. Rodriguez-Cabal, "Metaheuristic optimization methods for optimal power flow analysis in DC distribution networks," Transactions on Energy Systems and Engineering Applications, vol. 1, pp. 13-31, dec 2020.

[13] O. D. Montoya, A. Molina-Cabrera, H. R. Chamorro, L. AlvaradoBarrios, and E. Rivas-Trujillo, "A Hybrid Approach Based on SOCP and the Discrete Version of the SCA for Optimal Placement and Sizing DGs in AC Distribution Networks," Electronics, vol. 10, p. 26, dec 2020.

[14] S. Mirjalili, "SCA: A Sine Cosine Algorithm for solving optimization problems," Knowledge-Based Systems, vol. 96, pp. 120-133, mar 2016.

[15] H. Huang, X. Feng, A. A. Heidari, Y. Xu, M. Wang, G. Liang, H. Chen, and X. Cai, "Rationalized Sine Cosine Optimization With Efficient Searching Patterns," IEEE Access, vol. 8, pp. 61471-61490, 2020.

[16] A.-F. Attia, R. A. E. Sehiemy, and H. M. Hasanien, "Optimal power flow solution in power systems using a novel Sine-Cosine algorithm," Int. J. Electr. Power Energy Syst., vol. 99, pp. 331-343, jul 2018.

[17] J. A. Giraldo, O. D. Montoya, L. F. Grisales-Noreña, W. Gil-González, and M. Holguín, "Optimal power flow solution in direct current grids using Sine-Cosine algorithm,” J. Phys. Conf. Ser., vol. 1403, p. 012009 , nov 2019.

[18] A. I. Hafez, H. M. Zawbaa, E. Emary, and A. E. Hassanien, "Sine cosine optimization algorithm for feature selection," in 2016 International Symposium on INnovations in Intelligent SysTems and Applications (INISTA), IEEE, aug 2016.

[19] R. M. Rizk-Allah, "An improved sine-cosine algorithm based on orthogonal parallel information for global optimization," Soft Computing, vol. 23, pp. 7135-7161, jul 2018.

[20] S. Gupta, K. Deep, H. Moayedi, L. K. Foong, and A. Assad, "Sine cosine grey wolf optimizer to solve engineering design problems," Engineering with Computers, feb 2020.

[21] M. L. Manrique, O. D. Montoya, V. M. Garrido, L. F. Grisales-Noreña, and W. Gil-González, "Sine-Cosine Algorithm for OPF Analysis in Distribution Systems to Size Distributed Generators," in Communications in Computer and Information Science, pp. 28-39, Springer International Publishing, 2019.

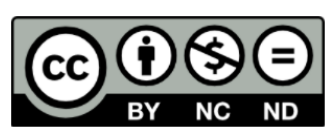

(C)2021 by the authors. Licensee TESEA, Cartagena, Colombia. This article is an open access article distributed under the terms and conditions of the Creative Commons Attribution (CC BY) license (http://creativecommons.org/licenses/by/4.0/ 